\title{
Single-stranded microRNA mimics
}

\author{
GUILLAUME CHORN, ${ }^{1,2}$ MOLLY KLEIN-MCDOWELL, ${ }^{1,3}$ LIHONG ZHAO, ${ }^{1,4}$ MATTHEW A. SAUNDERS, ${ }^{5}$ \\ W. MICHAEL FLANAGAN, ${ }^{6}$ AARRON T. WILLINGHAM, ${ }^{7}$ and LEE P. LIM ${ }^{8,9}$
}

Sirna Therapeutics, a wholly owned subsidiary of Merck \& Co., San Francisco, California 94158, USA

\begin{abstract}
miRNAs are 22 -nt RNAs that bind to the Argonaute family of proteins and have important regulatory roles in plants and animals. Here, we show that miRNAs exhibit targeting activity in cells when delivered as single strands that are 5 '-phosphorylated and that contain 2 '-fluoro ribose modifications. Length preferences, chemical modification sensitivity, and genome-wide seed-based targeting all suggest that this activity is Ago-based. Activity could be enhanced by annealing of segmented passenger strands containing non-nucleic acid spacers. Furthermore, screening of randomly generated sequences identified pyrimidine rich $3^{\prime}$ cassette sequences that increased single strand activity. These results provide an initial step in the development of single-stranded miRNA mimics for therapeutic use.
\end{abstract}

Keywords: miR-122; miR-124; microRNA; mimetic

\section{INTRODUCTION}

Shortly after the demonstration that double-stranded siRNAs could be designed to trigger RNA cleavage in mammalian cells (Elbashir et al. 2001), single-stranded, 5' -phosphorylated siRNAs were also found to induce RNAi, but at a greatly reduced potency (Martinez et al. 2002; Schwarz et al. 2002). Even though the incorporation of free, single-stranded RNA into Ago 2 is not known to be common in nature, singlestranded RNAi has potential as a therapeutic agent, especially given progress in the antisense field in the safe and effective dosing of single-stranded oligonucleotides (Raal et al. 2010).

Chemical modification has shown to be important for the therapeutic development of double-stranded siRNAs, resulting in increased nuclease stability, improved potency, reduced off-target effects, and elimination of immune stimulation (Bramsen and Kjems 2011). Work is also being done to chemically improve single-stranded siRNAs, with

\footnotetext{
${ }^{1}$ These authors contributed equally to this work.

${ }^{2}$ Present address: Aegis Advisors, Hong Kong, China

${ }^{3}$ Present address: University of Michigan Ross School of Business, Ann Arbor, MI 48109, USA

${ }^{4}$ Present address: Novartis, Emeryville, CA 94608, USA

${ }^{5}$ Present address: Sapphire Energy, San Diego, CA 92121, USA

${ }^{6}$ Present address: Genentech, South San Francisco, CA 94080, USA

${ }^{7}$ Present address: Merck Research Laboratory, Palo Alto, CA 94304, USA

${ }^{8}$ Present address: Department of Surgery, UCSF, San Francisco, CA 94143, USA

${ }^{9}$ Corresponding author

E-mail leeplim@gmail.com

Article published online ahead of print. Article and publication date are at http://www.rnajournal.org/cgi/doi/10.1261/rna.031278.111.
}

a recent paper showing that formulated, $2^{\prime}$-fluoro modified single-stranded siRNA has activity in vivo (Haringsma et al. 2012) and presentations showing that extensive chemical modification, including $5^{\prime}$ vinyl phosphonates, phosphorothioates, and 2 '-fluoro ribose, can allow subcutaneous delivery of unformulated single-stranded siRNAs with potency comparable to antisense oligonucleotides (Swayze et al. 2011).

microRNAs (miRNAs) are a class of $\sim 22$-nt noncoding RNAs that play roles in regulating gene expression in plants and animals. Precursor miRNA hairpins are usually cut by Dicer in the cytoplasm to produce a double-stranded duplex that is incorporated into Argonaute (Ago) proteins (Kim et al. 2009). After elimination of the passenger strand by cleavage and/or helicase activity, the guide strand binds complementary mRNAs. Studies suggest that the most prevalent form of consequential target recognition occurs through complementary binding to a target $3^{\prime}$ untranslated region (UTR) by the miRNA seed region (positions 2 through 8 at the $5^{\prime}$ end of the guide strand), leading to down-regulation of mRNA and protein levels (Bartel 2009).

While previous work analyzing single-stranded activity has focused on siRNAs, miRNAs also hold therapeutic promise, as restoration of miRNA levels is likely to be of benefit in maintaining tissue differentiation and inhibiting oncogenesis (Henry et al. 2011). Furthermore, the seedbased targeting of miRNAs allows for structures that would never be tolerated as siRNAs (Chorn et al. 2010), a characteristic that may be advantageous in optimizing miRNA single-strand mimics. We, therefore, decided to investigate 
whether chemically modified single strands can have miRNA activity and if there were ways to enhance that activity.

\section{RESULTS}

We investigated the effects of $5^{\prime}$-phosphorylation and $2^{\prime}$-fluoro modifications in the context of the microRNA miR124 , a brain-specific miRNA whose targeting we have studied extensively (Lim et al. 2005; Chorn et al. 2010) and that may have potential use in suppressing liver cancer (Hatziapostolou et al. 2011). We tested eight different 2 '-fluoro/2' ribo/ $/ 2^{\prime}$-o-methyl modification patterns, with or without a $5^{\prime}$ phosphate. Activity was assayed by measuring mRNA knockdown of two known miR-124 targets, CD164 (Fig. 1A) and VAMP3 (Supplemental Fig. S1A). Negligible activity in cell transfection assays was observed from miR-124 single strands containing 0 , 6, or 72 '-fluoros, while maximum single-stranded activity was observed from the patterns that contained both a $5^{\prime}$ phosphate and greater numbers $(12,13$, or 19) of $2^{\prime}$-fluoro modifications. Without the $5^{\prime}$ phosphate, only the pattern with 19 2'-fluoros ("21-8") had substantial activity. None of the singlestranded oligonucleotides was as potent as duplex miR-124, showing that there is substantial room for improvement of single-strand modification patterns.

We next tested a set of singlestranded, 5'-phosphorylated, 2'-fluoro oligos that all contained the miR-124 $5^{\prime}$ sequence but that varied in length from 10 to $22 \mathrm{nt}$ due to the addition of increasing amounts of the miR-124 3' sequence. Maximum activity was observed for lengths of 19 to $22 \mathrm{nt}$ (Fig. 1B), consistent with previous observations that miRNAs are of length 19 to 24 and consistent with the idea that the singlestranded miR-124 is acting through an Ago-dependent pathway.

We also tested the effect of substitution of a single $2^{\prime}$-o-methyl modification at every position along a $5^{\prime}$-phosphorylated, 2 '-fluoro 21-mer (Fig. 1C). Interestingly, substitution of a single $2^{\prime}$-fluoro by a 2 '-o-methyl had little effect on miR-124 activity, except for the substitution $2 \mathrm{nt}$
A

CD164 KD by miR-124 single strands

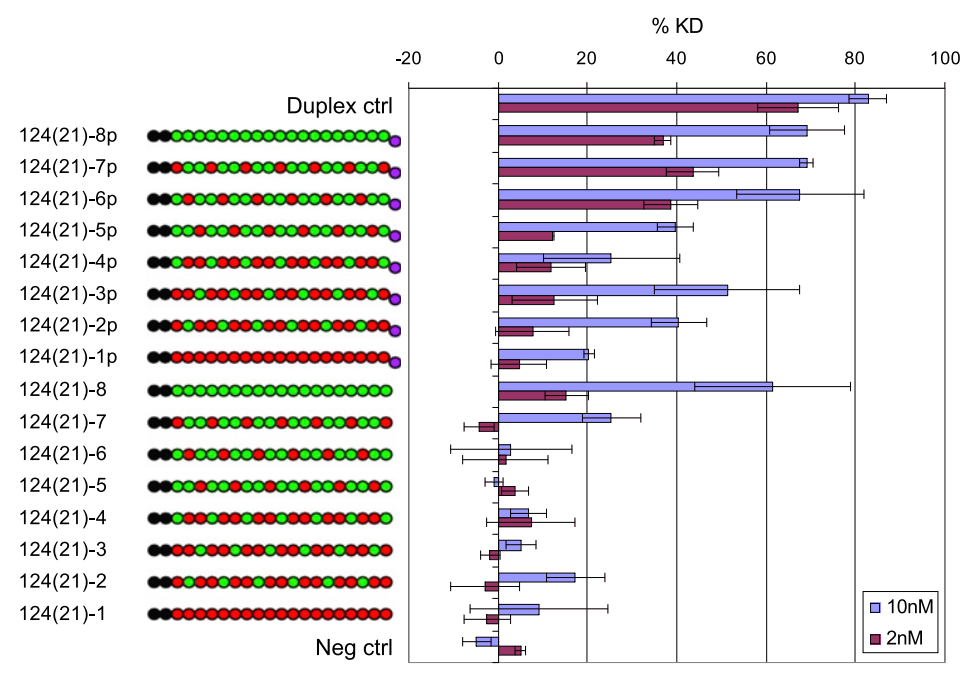

B

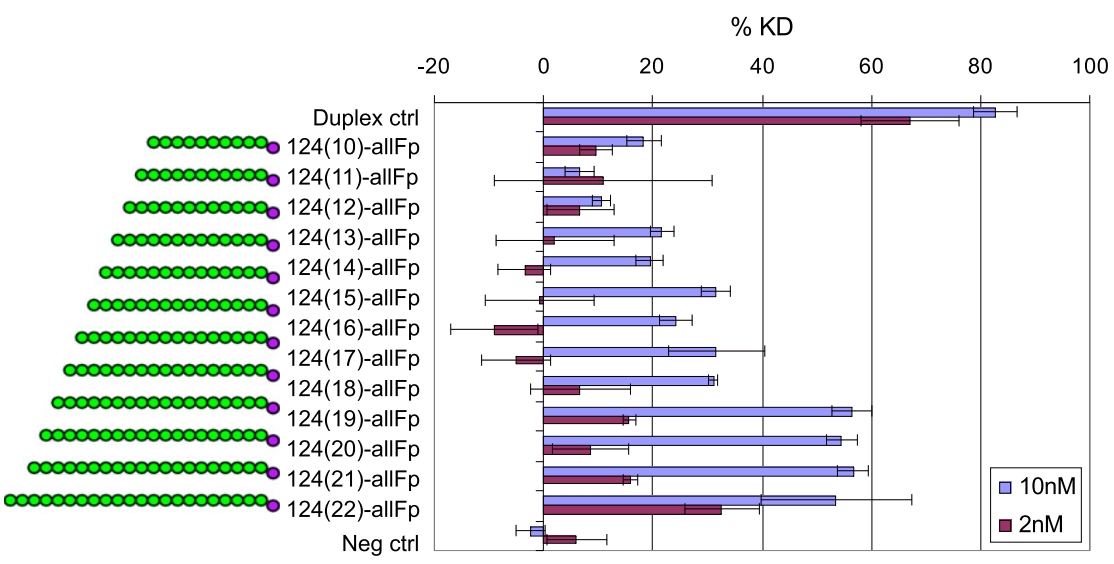

C

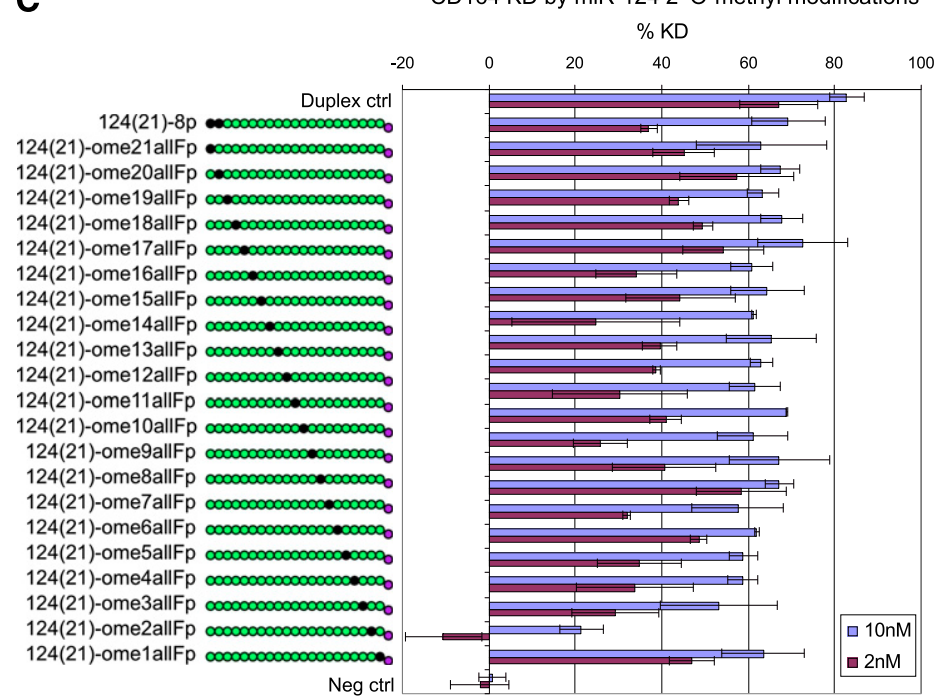

FIGURE 1. (Legend on next page) 
from the $5^{\prime}$ end, which led to a dramatic loss in potency. This result is in line with the previous observation that a $2^{\prime}$-o-methyl substitution at position 2 causes a significant reduction in Ago-based activity (Jackson et al. 2006). Furthermore, the fact that no other single substitution greatly decreases miR-124 activity suggests that the enhancement of single-strand activity is due to a distribution of $2^{\prime}$-fluoros across the transcript and not to one particular $2^{\prime}$-fluoro.

Given that miRNA activity often diminishes the transcript levels of many targets, we evaluated the activity of single-stranded miR-124 by transfecting cells and analyzing transcriptome-wide effects using a microarray (Lim et al. 2005). Analysis of the 3' UTRs of the down-regulated genes shows that the most significantly enriched hexamer and heptamer motifs are complementary matches to the miR-124 seed region (Fig. 2), providing objective evidence that singlestranded miR-124 carries out seed-based targeting, consistent with what is known about Ago-based miRNA activity.

Despite the fact that a chemically modified single-stranded miR-124 has clear seed-based activity, it is still not as potent as a duplex: For example, the addition of a passenger strand to the 21-8p guide strand leads to a substantial increase in activity (Fig. 3). We, therefore, sought to determine if activity could be regained by adding back portions of the passenger strand to an active single strand, with the eventual goal of defining minimal structures for future studies of miRNA mimics. We first tested the effects of adding back either a $5^{\prime}$ or $3^{\prime}$ fragment of the passenger strand. This type of structure was tested previously in the context of an siRNA with a predominantly RNA guide strand (Bramsen et al. 2007) in a study that observed activity only when both passenger fragments were annealed to the guide strand and not when one or none of the passenger fragments was annealed. In this paper, since $21-8 \mathrm{p}$ has activity by itself, we are able to detect effects of the passenger fragments on the guide strand's baseline activity. Interestingly, annealing of either the $5^{\prime}$ fragment (P9, which forms $9 \mathrm{bp}$ ) or the $3^{\prime}$ fragment (P.10, which forms 8 bp and a 2-base overhang) led to a significant decrease of activity relative to the original $21-8 \mathrm{p}$ single strand (Fig. 3). In contrast, annealing both fragments at once led to a dramatic increase in activity, close to that observed after annealing a full-length passenger strand. These results suggest that both passenger fragments are able to bind the guide strand individually but must be bound simultaneously if they are to enhance, and not impede, activity.

\section{HCT-116 cells transfected with 124(21)-8p}

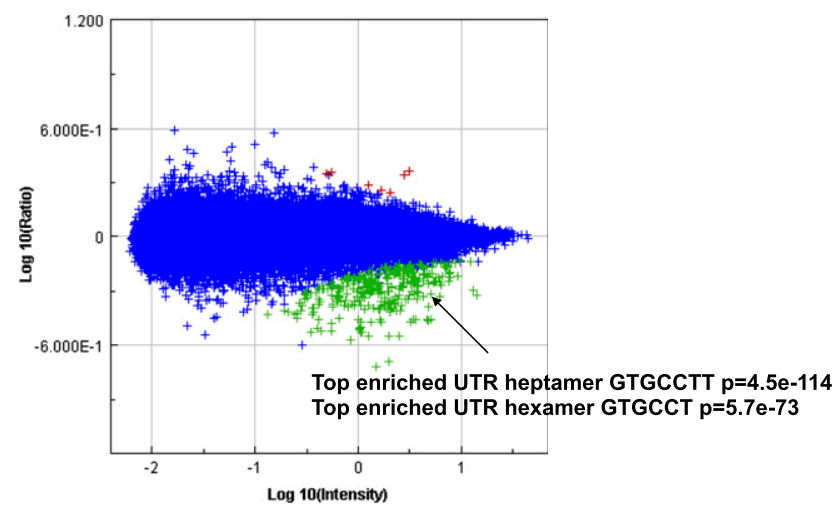

FIGURE 2. Microarray analysis shows seed-based activity from a miR-124 single strand. Microarray signature $24 \mathrm{~h}$ after transfection of $10 \mathrm{nM}$ miR-124 21-8p into HCT116 cells, plotted as ratio of fluorescence intensity (relative to mock transfection) on the $y$-axis and mean fluorescence intensity for the experimental and mock samples on the $x$-axis. Significantly down-regulated probes $\left(P<1 \times 10^{6}\right)$ are shown in green and significantly up-regulated probes in red. Hypergeometric analysis of the hexamer and heptamer content of the downregulated 3' UTRs showed that the most significantly enriched heptamers and hexamers are GTGCCTT and GTGCCT, respectively.

To follow up on these results, we added 6,7 , or 8 bp of complementarity simultaneously to both ends of the guide strand, connecting the $5^{\prime}$ and $3^{\prime}$ binding segments with a linker segment consisting of carbon spacers to improve binding affinity (Fig. 4). The addition of the passenger strand that created 8 bp of double-stranded nucleic acid at both ends of the guide strand $(\mathrm{P} 8.8 \mathrm{c} 3)$ resulted in a significant increase in potency compared with the guide strand alone, though it was still not as active as the fully duplexed guide strand. Conversely, the addition of a passenger strand with 6- or 7-bp ends (P6.6c3 and P7.7c3, respectively) had either negligible or negative effects when compared to the single-strand guide strand by itself. Given our previous results, this may be a result of loss of binding at one end or another due to the small regions of complementarity; we, therefore, added locked nucleic acid (LNA) bases to increase binding affinity (Bramsen et al. 2007). The addition of LNAs did, in fact, improve the activity of P6.6c3 and P7.7c3, with the P7.7c3-2.2LNA having similar activity to $\mathrm{P} 8.8 \mathrm{c} 3$.

The seed-based activity of miRNAs provides the opportunity to study the influence of the base composition of the $3^{\prime}$ half of the single strand on activity without making major changes to target site complementarity. We, therefore, designed single-strand oligos that shared the miR-124 21-8p seed sequence and chemical modification pattern but in which bases 10 through 19 were randomly generated. Eightyeight sequences were synthesized, after binning into 11 different sets of eight 


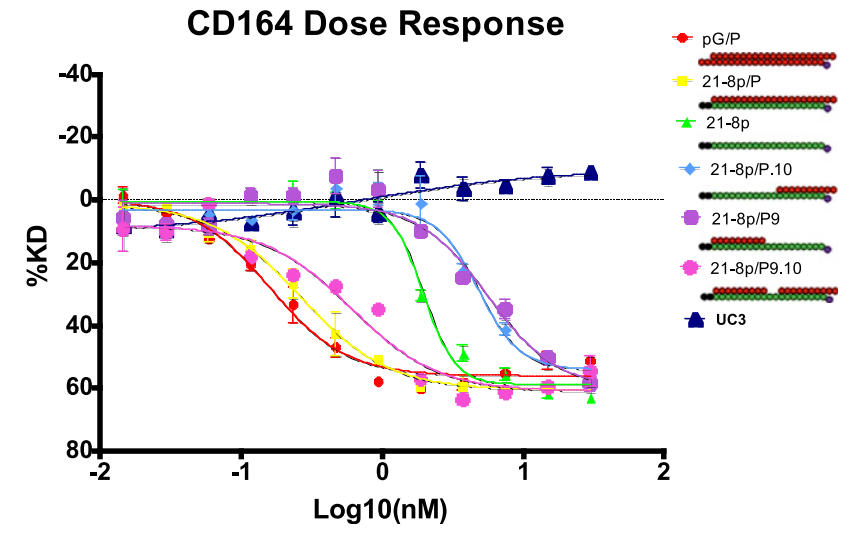

FIGURE 3. Activity of the fluorinated and phosphorylated miR-124 single strand is enhanced by the addition of $5^{\prime}$ and $3^{\prime}$ passenger fragments, but either fragment alone decreases activity. Dose-dependent response of CD164 mRNA in HCT-116 cells, as measured by qRT-PCR. Schematic of annealed strands depicts modified strands and passenger segments, where $2^{\prime}-\mathrm{OH}$ is denoted in red, $2^{\prime}$-fluoro is green, $2^{\prime}$-o-methyl is black, and $5^{\prime}$ phosphate is purple.

based on pyrimidine content (i.e., 0 to 10 pyrimidines). The activity of each oligo was tested against miR-124 targets CD164 and VAMP3 (Fig. 5A; Supplemental Fig. S4), and several sequences showed greater activity than the original miR-124 sequence. Importantly, a significant positive correlation was observed between pyrimidine content of the random cassette and activity (correlation $=0.64, P$-val $=$ $\left.2 \times 10^{11}\right)$. A sequence logo of the top quartile performers (Fig. 5B) clearly shows this enrichment for pyrimidines in the highest performing oligos, suggesting that pyrimidines are more conducive to single-strand activity than purines, at least in the context of a highly $2^{\prime}$-fluoro single strand. Nevertheless, it is worth noting that not every high pyrimidine cassette was a top performer. Further experiments will likely be required to understand the reasons for this.

We also tested whether a $3^{\prime}$ cassette sequence that had been identified in the context of a miR-124 seed could be of use when fused to the seed region of a different miRNA, the liver-specific miR-122. Perhaps because of its relatively low pyrimidine content, the original miR-122 sequence had little activity as a fluorinated single strand against the miR122 target GYS1, especially when compared with a duplex miR-122 (Fig. 5C). However, when the $3^{\prime}$ miR-122 sequence was replaced with one of the high performing cassettes ("9Y_2," containing 9 pyrimidines), targeting activity was greatly enhanced, both against GYS1 (Fig. 5C) and SLC7A1 (Supplemental Fig. S5). These data make it unlikely that the enhanced activity is due to serendipitous pairing of the cassette region to the target site, given that the same cassette improved activity against four different mRNA targets (of two different miRNAs). Furthermore, analysis of the target site sequences showed no evidence for additional pairing from the cassette sequence being responsible for the improved activity (Supplemental Fig. S6).

\section{DISCUSSION}

Our results show that $5^{\prime}$-phosphorylated, $2^{\prime}$-fluoro modified oligonucleotides exhibit substantial miRNA seed-based activity in cells. Although we have not shown direct biochemical evidence that this is an Ago-mediated process, the observation of pervasive seed-based activity suggests that these strands are being incorporated into Ago proteins. Furthermore, the length preference of 19 to $22 \mathrm{nt}$, as well as the sensitivity to modification at position 2 by a 2 '-o-methyl, are also indicators of Ago-based activity.

Since even a fully 2 '-fluoro modified single strand had less activity than duplex RNA, it will be important to find ways to further improve the potency of single-stranded miRNA mimics while still maintaining some of its singlestrand nature. One method tried here was the annealing of passenger strand fragments to the guide strand. Duplexes of 6,7 , and $8 \mathrm{bp}$ at both ends of the guide strand (coupled with a linker between the fragments and addition of LNA modifications to the 6- or 7-bp fragments) were found to improve activity. Previous observations have found that shorter passenger strands forming around 14 contiguous base pairs of duplex with a guide strand have significant siRNA activity (Chu and Rana 2008; Sun et al. 2008). Our results agree with these findings and extend them by showing that the duplex portions can be separated by extensive gaps. The toleration of these gaps may improve delivery of miRNA mimics by reducing overall mass and charge and allowing the insertion of chemical moieties that enhance delivery or Ago activity. It will also be interesting to see if it is possible to create even smaller duplex structures at the very ends of the single strand that lead to increased activity.

On the other hand, we were surprised to observe that the asymmetric formation of 8 or 9 bp of duplex at the $5^{\prime}$ or $3^{\prime}$

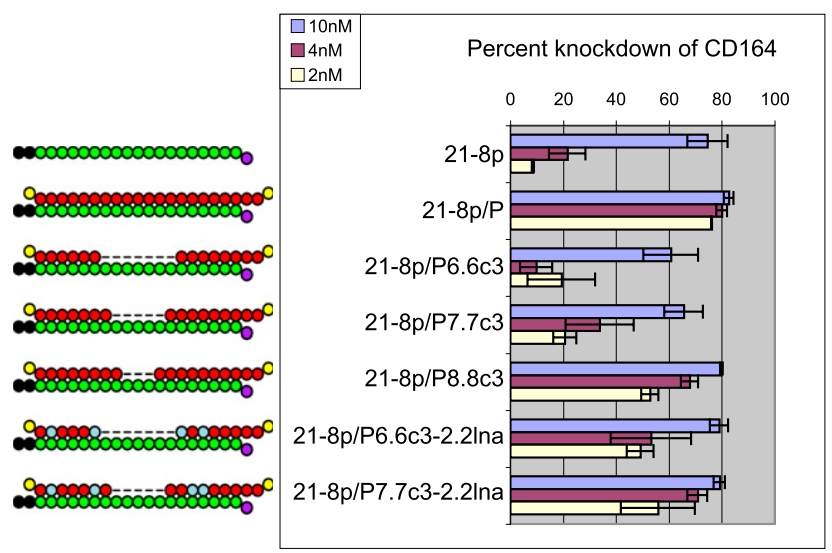

FIGURE 4. Restoration of activity by addition of $3^{\prime}$ and $5^{\prime}$ passenger strand fragments linked by carbon spacers. HCT-116 cells were transfected at $10 \mathrm{nM}, 4 \mathrm{nM}$, and $2 \mathrm{nM}$, and CD164 mRNA was measured by qRT-PCR. $2^{\prime}-\mathrm{OH}$ is depicted in red, $2^{\prime}$-fluoro in green, $2^{\prime}$-o-methyl in black, LNA in light blue, $5^{\prime}$ phosphate in purple, and inverted abasic in yellow. The C3-spacer (ChemGenes CLP-9908) is represented by a dash. 


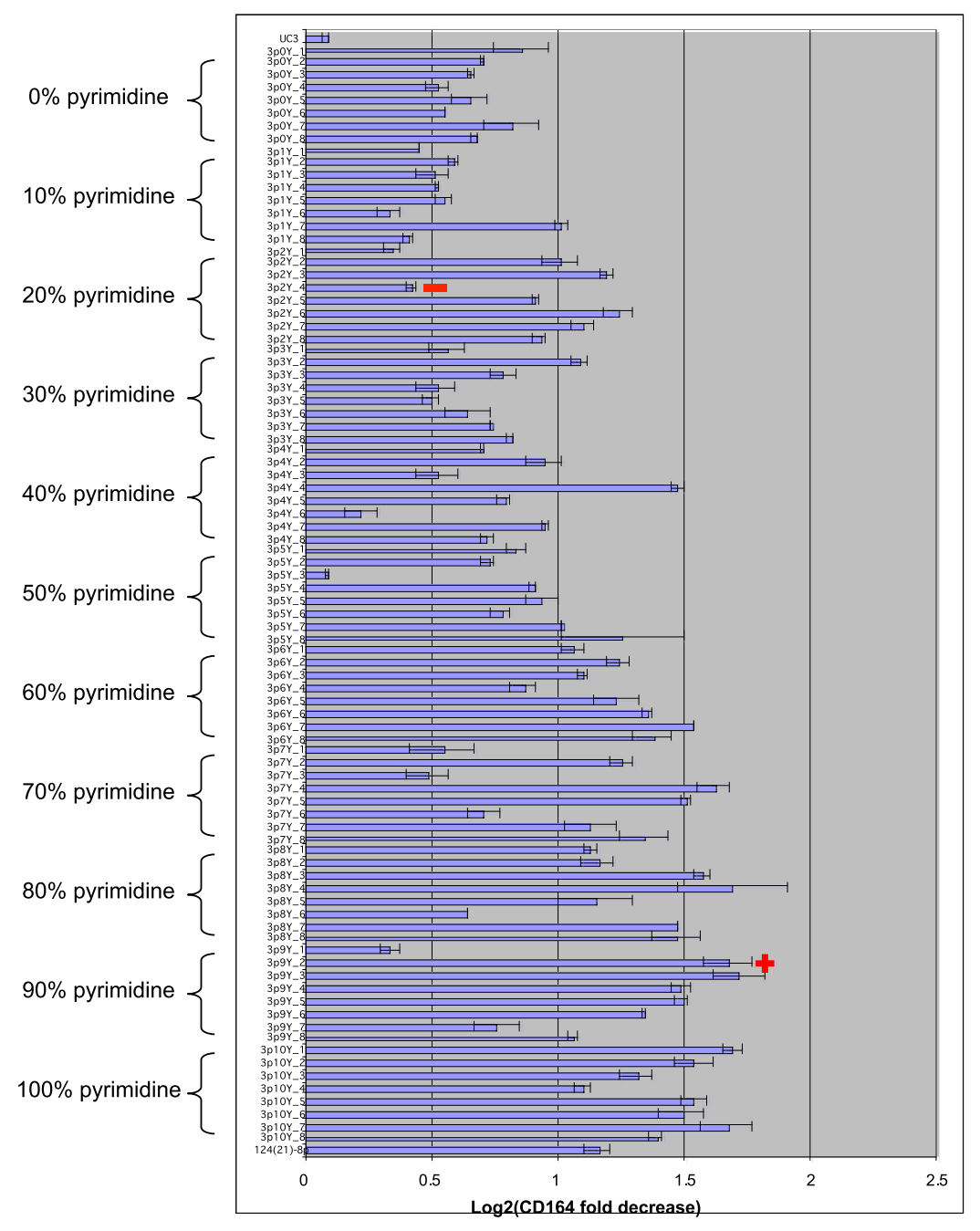

A Testing of 3' ends with differing levels of pyrimidine composition demonstrates importance of sequence composition on single strand activity

- “2Y_4”, low performer

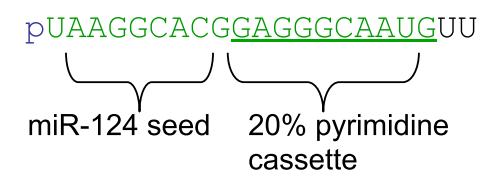

"9Y_2”, high performer

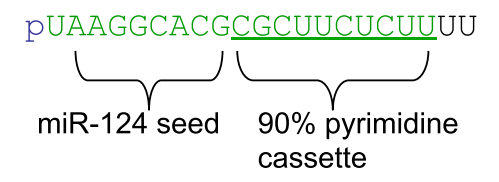

B Sequence composition of top quartile performers.

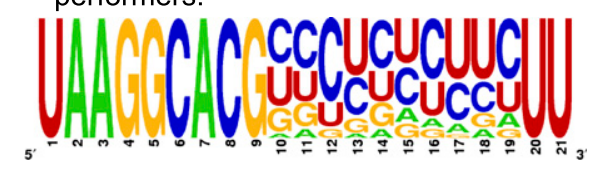

C Knockdown of miR-122 target GYS1

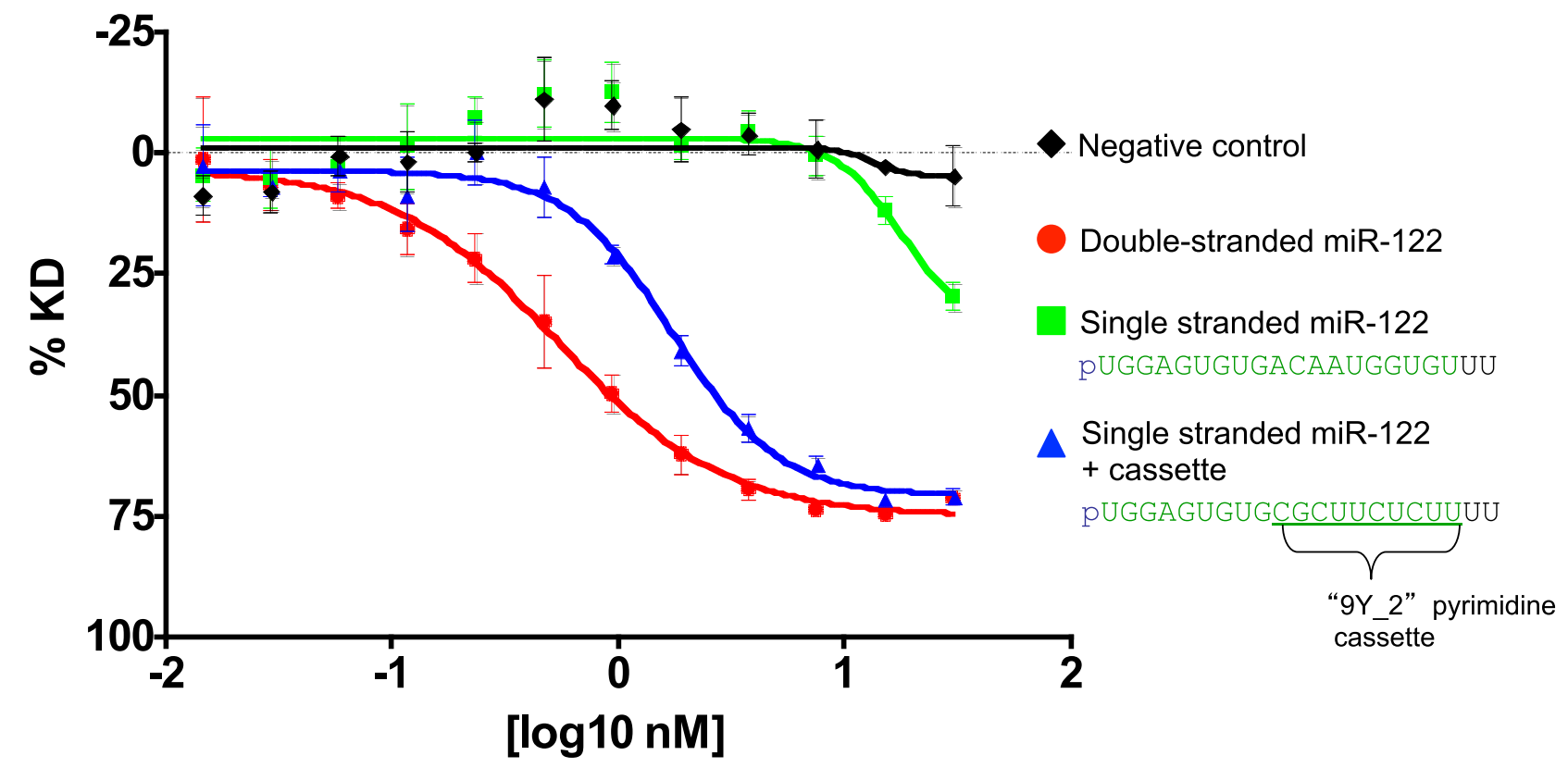

FIGURE 5. Analysis of randomly selected 3' sequences in the context of the " $21-8 \mathrm{p}$ " modification pattern. (A) Activity of miR-124 single strands with randomized bases at positions 10 through 19. CD164 mRNA levels were measured by qRT-PCR following transfection of HCT-116 cells at 10 nM. (B) Sequence logo of miR-124 single strands with activity in the top quartile. (C) Pyrimidine-rich 3' cassette can enhance single-strand miR122 activity. Dose response curve of miR-122 target GYS1 to increasing amounts of the indicated oligos. (Red) miR-122 control duplex, (black) nontargeting control siRNA, (green) miR-122 sequence in the " $21-8 \mathrm{p}$ " modification pattern, and (blue) miR-122 seed sequence fused with the "9Y_2" pyrimidine-rich 3 ' cassette sequence, also in the $21-8 \mathrm{p}$ modification pattern. 
end of the guide strand, respectively, led to a decrease in activity relative to the original single strand. It is possible that the pathways for single-stranded and double-stranded incorporation into Ago are distinct and that a structure in which one end is single-stranded and one end is doublestranded prevents productive entry into either. Alternatively, perhaps an 8- or 9-bp passenger fragment is unable to be released following incorporation into the RISC, while longer passenger strands can be detected by the RISC and cleaved and/or removed. Obviously, more biochemical approaches will be required to understand this behavior.

By randomizing the $3^{\prime}$ sequence of single-stranded miR124 , we were also able to identify sequences that promoted its activity. These sequences tended to contain a high percentage of pyrimidines, which may reflect a positive effect of pyrimidines or a negative effect of purines. Importantly, we found that replacing 10 bases of the $3^{\prime}$ sequence of a miR-122 single strand with a pyrimidine-rich cassette sequence greatly enhanced its potency, showing that $3^{\prime}$ sequence optimization could be a powerful tool in developing miRNA mimics. Understanding the reasons why certain sequences work well in this context will be an interesting area for further study.

We have little understanding of why 2 '-fluoro content and pyrimidine content have such beneficial effects on the activity of single strands. The $2^{\prime}$-fluoro modification's unique electronegativity helps to maintain a C3'-endo conformation and gives oligonucleotides novel hydrophobic properties and basestacking properties (Manoharan et al. 2011). It is possible that 2 '-fluoro content and certain pyrimidine-rich sequences help to put single strands into a conformation or hydration state that facilitates Ago binding and enhances stability.

These studies provide an initial step in the development of single-stranded miRNA mimetics and suggest that their effectiveness can be improved by using certain sequences and modification patterns. Addition of further chemical modifications such as phosphorothioates should allow for the in vivo delivery of nonformulated single strands, providing a way to dose miRNA mimics without the complications of delivery vehicles.

\section{MATERIALS AND METHODS}

\section{Oligonucleotide synthesis}

Oligonucleotides were synthesized at Sigma or Merck \& Co. using standard solid-phase synthesis followed by purification by ionexchange chromatography. Passenger strands were annealed by combining oligonucleotides at $10 \mu \mathrm{M}$ in $10 \mathrm{mM} \mathrm{pH7.5} \mathrm{Tris-HCl/}$ $50 \mathrm{mM} \mathrm{NaCl}$ and heating at $95^{\circ} \mathrm{C}$ for $2 \mathrm{~min}$, followed by gradual cooling for $1 \mathrm{~h}$ to $37^{\circ} \mathrm{C}$.

\section{qPCR measurement}

HCT-116 cells, cultured in McCoy's 5A Medium, supplemented with $10 \%$ fetal bovine serum and $1 \%$ penicillin-streptomycin, and plated at a density of 6000 cells/well in 96-well culture plates
$24 \mathrm{~h}$ prior to transfection, were transfected with Lipofectamine RNAiMax (Invitrogen) and Opti-MEM I Reduced Serum Media (GIBCO). Twenty-four hours post-transfection, cells were rinsed with phosphate-buffered saline and processed with the Cells-toCT Kit (Applied Biosystems). TaqMan gene-specific probes were used on an ABI Prism 9700HT Sequence Detector for RT-qPCR. Reverse transcription conditions were as follows: $2 \mathrm{~min}$ at $50^{\circ} \mathrm{C}$, $10 \mathrm{~min}$ at $95^{\circ} \mathrm{C}$, followed by 40 cycles of $15 \mathrm{sec}$ at $95^{\circ} \mathrm{C}$, and $1 \mathrm{~min}$ at $60^{\circ} \mathrm{C}$. GUSB mRNA levels were used for data normalization. Knockdown was calculated as $\Delta \mathrm{Ct}=\mathrm{Ct}_{\text {Target }}-\mathrm{Ct}_{\mathrm{GUSB}}$ and $\Delta \Delta \mathrm{Ct}=$ $\Delta \mathrm{Ct}_{(\exp )}-\Delta \mathrm{Ct}_{(\mathrm{ctrl})}$ and relative expression level $=2^{-\Delta \Delta \mathrm{Ct}}$ and $\%$ knockdown $=100 \times\left(1-2^{-\Delta \Delta C t}\right)$.

\section{Microarray and bioinformatics analysis}

HCT-116 cells were transfected with $10 \mathrm{nM}$ miRNA as described previously (Jackson et al. 2006). RNA was extracted using RNeasy (Qiagen), amplified using the Ovation protocol (Nugen), and profiled on custom Affymetrix arrays (Rosetta Custom Human 2.0, Affymetrix). Array signals were analyzed with Affymetrix GeneChip Operating Software and Affymetrix Power Tools. UTR hexamer and heptamer enrichment was analyzed using the hypergeometric distribution as described previously (Jackson et al. 2006). Sequence logo was generated using WebLogo (Crooks et al. 2004).

\section{Oligonucleotide sequences}

Oligonucleotides tested are listed below $\left(5^{\prime}\right.$ to $3^{\prime}, \mathrm{r}=$ ribo, ome $=$ $2^{\prime}$-o-methyl, flu = 2' -fluoro, $\mathrm{iB}=$ inverted abasic, $\mathrm{d}=$ deoxy, $\operatorname{lna}=$ locked nucleic acid, $\mathrm{c} 3=$ propane diol spacer, $p=5^{\prime}$ phosphate).

124(21)-1 rU;rA;rA;rG;rG;rC;rA;rC;rG;rC;rG;rG;rU;rG;rA;rA;rU; rG;rC;omeC;omeA

124(21)-2 rU;rA;fluA;rG;rG;fluC;rA;rC;fluG;rC;rG;fluG;rU;rG; fluA;rA;rU;fluG;rC;omeC;omeA

124(21)-3 rU;fluA;rA;rG;fluG;rC;rA;fluC;rG;rC;fluG;rG;rU;fluG; rA;rA;fluU;rG;rC;omeC;omeA

124(21)-4 fluU;rA;rA;fluG;rG;rC;fluA;rC;rG;fluC;rG;rG;fluU;rG; rA;fluA;rU;rG;fluC;omeC;omeA

124(21)-5 fluU;rA;fluA;fluG;rG;fluC;fluA;rC;fluG;fluC;rG;fluG; fluU;rG;fluA;fluA;rU;fluG;fluC;omeC;omeA

124(21)-6 fluU;fluA;rA;fluG;fluG;rC;fluA;fluC;rG;fluC;fluG;rG; fluU;fluG;rA;fluA;fluU;rG;fluC;omeC;omeA

124(21)-7 rU;fluA;fluA;rG;fluG;fluC;rA;fluC;fluG;rC;fluG;fluG; rU;fluG;fluA;rA;fluU;fluG;rC;omeC;omeA

124(21)-8 fluU;fluA;fluA;fluG;fluG;fluC;fluA;fluC;fluG;fluC;fluG; fluG;fluU;fluG;fluA;fluA;fluU;fluG;fluC;omeC;omeA

124(21)-1p p;rU;rA;rA;rG;rG;rC;rA;rC;rG;rC;rG;rG;rU;rG;rA;rA; rU;rG;rC;omeC;omeA

124(21)-2p p;rU;rA;fluA;rG;rG;fluC;rA;rC;fluG;rC;rG;fluG;rU;rG; fluA;rA;rU;fluG;rC;omeC;omeA

124(21)-3p p;rU;fluA;rA;rG;fluG;rC;rA;fluC;rG;rC;fluG;rG;rU;fluG; rA;rA;fluU;rG;rC;omeC;omeA

124(21)-4p p;fluU;rA;rA;fluG;rG;rC;fluA;rC;rG;fluC;rG;rG;fluU; rG;rA;fluA;rU;rG;fluC;omeC;omeA

124(21)-5p p;fluU;rA;fluA;fluG;rG;fluC;fluA;rC;fluG;fluC;rG;fluG; fluU;rG;fluA;fluA;rU;fluG;fluC;omeC;omeA

124(21)-6p p;fluU;fluA;rA;fluG;fluG;rC;fluA;fluC;rG;fluC;fluG;rG; fluU;fluG;rA;fluA;fluU;rG;fluC;omeC;omeA 


\section{Chorn et al.}

124(21)-7p p;rU;fluA;fluA;rG;fluG;fluC;rA;fluC;fluG;rC;fluG;fluG; rU;fluG;fluA;rA;fluU;fluG;rC;omeC;omeA

124(21)-8p p;fluU;fluA;fluA;fluG;fluG;fluC;fluA;fluC;fluG;fluC; fluG;fluG;fluU;fluG;fluA;fluA;fluU;fluG;fluC;omeC;omeA

miR-124 duplex ctrl iB;rG;rC;rA;rU;rU;rC;rA;rC;rC;rG;rC;rG;rU; rG;rC;rC;rU;rU;rA;dA;dT;iB rU;rA;rA;rG;rG;rC;rA;rC;rG;rC;rG; rG;rU;rG;rA;rA;rU;rG;rC;omeC;omeA

124(22)-allFp p;fluU;fluA;fluA;fluG;fluG;fluC;fluA;fluC;fluG;fluC; fluG;fluG;fluU;fluG;fluA;fluA;fluU;fluG;fluC;fluA;fluC;fluA

124(21)-allFp p;fluU;fluA;fluA;fluG;fluG;fluC;fluA;fluC;fluG;fluC; fluG;fluG;fluU;fluG;fluA;fluA;fluU;fluG;fluC;fluC;fluA

124(20)-allFp p;fluU;fluA;fluA;fluG;fluG;fluC;fluA;fluC;fluG;fluC; fluG;fluG;fluU;fluG;fluA;fluA;fluU;fluG;fluC;fluA

124(19)-allFp p;fluU;fluA;fluA;fluG;fluG;fluC;fluA;fluC;fluG;fluC; fluG;fluG;fluU;fluG;fluA;fluA;fluU;fluC;fluA

124(18)-allFp p;fluU;fluA;fluA;fluG;fluG;fluC;fluA;fluC;fluG;fluC; fluG;fluG;fluU;fluG;fluA;fluA;fluC;fluA

124(17)-allFp p;fluU;fluA;fluA;fluG;fluG;fluC;fluA;fluC;fluG;fluC; fluG;fluG;fluU;fluG;fluA;fluC;fluA

124(16)-allFp p;fluU;fluA;fluA;fluG;fluG;fluC;fluA;fluC;fluG;fluC; fluG;fluG;fluU;fluG;fluC;fluA

124(15)-allFp p;fluU;fluA;fluA;fluG;fluG;fluC;fluA;fluC;fluG;fluC; fluG;fluG;fluU;fluC;fluA

124(14)-allFp p;fluU;fluA;fluA;fluG;fluG;fluC;fluA;fluC;fluG;fluC; fluG;fluG;fluC;fluA

124(13)-allFp p;fluU;fluA;fluA;fluG;fluG;fluC;fluA;fluC;fluG;fluC; fluG;fluC;fluA

124(12)-allFp p;fluU;fluA;fluA;fluG;fluG;fluC;fluA;fluC;fluG;fluC; fluC;fluA

124(11)-allFp p;fluU;fluA;fluA;fluG;fluG;fluC;fluA;fluC;fluG;fluC;fluA 124(10)-allFp p;fluU;fluA;fluA;fluG;fluG;fluC;fluA;fluC;fluC;fluA

124(21)-ome1allFp p;omeU;fluA;fluA;fluG;fluG;fluC;fluA;fluC;fluG; fluC;fluG;fluG;fluU;fluG;fluA;fluA;fluU;fluG;fluC;fluC;fluA

124(21)-ome2allFp p;fluU;omeA;fluA;fluG;fluG;fluC;fluA;fluC;fluG; fluC;fluG;fluG;fluU;fluG;fluA;fluA;fluU;fluG;fluC;fluC;fluA

124(21)-ome3allFp p;fluU;fluA;omeA;fluG;fluG;fluC;fluA;fluC;fluG; fluC;fluG;fluG;fluU;fluG;fluA;fluA;fluU;fluG;fluC;fluC;fluA

124(21)-ome4allFp p;fluU;fluA;fluA;omeG;fluG;fluC;fluA;fluC;fluG; fluC;fluG;fluG;fluU;fluG;fluA;fluA;fluU;fluG;fluC;fluC;fluA

124(21)-ome5allFp p;fluU;fluA;fluA;fluG;omeG;fluC;fluA;fluC;fluG; fluC;fluG;fluG;fluU;fluG;fluA;fluA;fluU;fluG;fluC;fluC;fluA 124(21)-ome6allFp p;fluU;fluA;fluA;fluG;fluG;omeC;fluA;fluC;fluG; fluC;fluG;fluG;fluU;fluG;fluA;fluA;fluU;fluG;fluC;fluC;fluA

124(21)-ome7allFp p;fluU;fluA;fluA;fluG;fluG;fluC;omeA;fluC;fluG; fluC;fluG;fluG;fluU;fluG;fluA;fluA;fluU;fluG;fluC;fluC;fluA

124(21)-ome8allFp p;fluU;fluA;fluA;fluG;fluG;fluC;fluA;omeC;fluG; fluC;fluG;fluG;fluU;fluG;fluA;fluA;fluU;fluG;fluC;fluC;fluA

124(21)-ome9allFp p;fluU;fluA;fluA;fluG;fluG;fluC;fluA;fluC;omeG; fluC;fluG;fluG;fluU;fluG;fluA;fluA;fluU;fluG;fluC;fluC;fluA

124(21)-ome10allFp p;fluU;fluA;fluA;fluG;fluG;fluC;fluA;fluC;fluG; omeC;fluG;fluG;fluU;fluG;fluA;fluA;fluU;fluG;fluC;fluC;fluA

124(21)-ome11allFp p;fluU;fluA;fluA;fluG;fluG;fluC;fluA;fluC;fluG; fluC;omeG;fluG;fluU;fluG;fluA;fluA;fluU;fluG;fluC;fluC;fluA

124(21)-ome12allFp p;fluU;fluA;fluA;fluG;fluG;fluC;fluA;fluC;fluG; fluC;fluG;omeG;fluU;fluG;fluA;fluA;fluU;fluG;fluC;fluC;fluA 124(21)-ome13allFp p;fluU;fluA;fluA;fluG;fluG;fluC;fluA;fluC;fluG; fluC;fluG;fluG;omeU;fluG;fluA;fluA;fluU;fluG;fluC;fluC;fluA

124(21)-ome14allFp p;fluU;fluA;fluA;fluG;fluG;fluC;fluA;fluC;fluG; fluC;fluG;fluG;fluU;omeG;fluA;fluA;fluU;fluG;fluC;fluC;fluA
124(21)-ome15allFp p;fluU;fluA;fluA;fluG;fluG;fluC;fluA;fluC;fluG; fluC;fluG;fluG;fluU;fluG;omeA;fluA;fluU;fluG;fluC;fluC;fluA

124(21)-ome16allFp p;fluU;fluA;fluA;fluG;fluG;fluC;fluA;fluC;fluG; fluC;fluG;fluG;fluU;fluG;fluA;omeA;fluU;fluG;fluC;fluC;fluA

124(21)-ome17allFp p;fluU;fluA;fluA;fluG;fluG;fluC;fluA;fluC;fluG; fluC;fluG;fluG;fluU;fluG;fluA;fluA;omeU;fluG;fluC;fluC;fluA

124(21)-ome18allFp p;fluU;fluA;fluA;fluG;fluG;fluC;fluA;fluC;fluG; fluC;fluG;fluG;fluU;fluG;fluA;fluA;fluU;omeG;fluC;fluC;fluA

124(21)-ome19allFp p;fluU;fluA;fluA;fluG;fluG;fluC;fluA;fluC;fluG; fluC;fluG;fluG;fluU;fluG;fluA;fluA;fluU;fluG;omeC;fluC;fluA

124(21)-ome20allFp p;fluU;fluA;fluA;fluG;fluG;fluC;fluA;fluC;fluG; fluC;fluG;fluG;fluU;fluG;fluA;fluA;fluU;fluG;fluC;omeC;fluA

124(21)-ome21allFp p;fluU;fluA;fluA;fluG;fluG;fluC;fluA;fluC;fluG; fluC;fluG;fluG;fluU;fluG;fluA;fluA;fluU;fluG;fluC;fluC;omeA

P9 rG;rC;rA;rU;rU;rC;rA;rC;rC

P.10 rG;rU;rG;rC;rC;rU;rU;rA;rA;rU

P rG;rC;rA;rU;rU;rC;rA;rC;rC;rG;rC;rG;rU;rG;rC;rC;rU;rU;rA; $\mathrm{rA} ; \mathrm{rU}$

pG p;rU;rA;rA;rG;rG;rC;rA;rC;rG;rC;rG;rG;rU;rG;rA;rA;rU;rG;rC; omeC;omeA

iPi iB;rG;rC;rA;rU;rU;rC;rA;rC;rC;rG;rC;rG;rU;rG;rC;rC;rU;rU; $\mathrm{rA} ; \mathrm{rA} ; \mathrm{rU} ; \mathrm{iB}$

P8.8c3 iB;rG;rC;rA;rU;rU;rC;rA;rC;c3;c3;c3;rG;rU;rG;rC;rC;rU; rU;rA;rA;rU;iB

P7.7c3 iB;rG;rC;rA;rU;rU;rC;rA;c3;c3;c3;c3;c3;rU;rG;rC;rC;rU;rU; $\mathrm{rA} ; \mathrm{rA} ; \mathrm{rU} ; \mathrm{iB}$

P7.7c3-2.2lna iB;rG;lnaC;rA;rU;rU;lnaC;rA;c3;c3;c3;c3;c3;rU;rG; $\operatorname{lnaC} ; \operatorname{lnaC} ; \mathrm{rU} ; \mathrm{rU} ; \mathrm{rA} ; \mathrm{rA} ; \mathrm{rU} ; \mathrm{i} / 45 \mathrm{~B}$

P6.6c3 iB;rG;rC;rA;rU;rU;rC;c3;c3;c3;c3;c3;rG;rC;rC;rU;rU;rA;rA; $\mathrm{rU} ; \mathrm{iB}$

P6.6c3-2.2lna iB;rG;lnaC;rA;rU;rU;lnaC;c3;c3;c3;c3;c3;lnaG;rC; lnaC;rU;rU;rA;rA;rU;iB

miR-122 duplex ctrl iB;rA;rC;rA;rC;rC;rA;rU;rU;rG;rU;rC;rA;rC; rA;rC;rU;rC;rC;rA;omeA;omeU;iBrU;rG;rG;rA;rG;rU;rG;rU; rG;rA;rC;rA;rA;rU;rG;rG;rU;rG;rU;omeU;omeU

mir122-8p p;fluU;fluG;fluG;fluA;fluG;fluU;fluG;fluU;fluG;fluA; fluC;fluA;fluA;fluU;fluG;fluG;fluU;fluG;fluU;omeU;omeU

mir122-9Y_2-8p p;fluU;fluG;fluG;fluA;fluG;fluU;fluG;fluU;fluG; fluC;fluG;fluC;fluU;fluU;fluC;fluU;fluC;fluU;fluU;omeU;omeU

3p0Y_1 p;fluU;fluA;fluA;fluG;fluG;fluC;fluA;fluC;fluG;fluA;fluG; fluG;fluA;fluG;fluG;fluA;fluA;fluG;fluA;omeU;omeU

3p0Y_2 p;fluU;fluA;fluA;fluG;fluG;fluC;fluA;fluC;fluG;fluA;fluG; fluG;fluA;fluA;fluG;fluG;fluA;fluA;fluG;omeU;omeU

3p0Y_3 p;fluU;fluA;fluA;fluG;fluG;fluC;fluA;fluC;fluG;fluG;fluA; fluA;fluG;fluG;fluA;fluA;fluA;fluG;fluG;omeU;omeU

3p0Y_4 p;fluU;fluA;fluA;fluG;fluG;fluC;fluA;fluC;fluG;fluA;fluA; fluG;fluA;fluG;fluG;fluG;fluA;fluA;fluG;omeU;omeU

3p0Y_5 p;fluU;fluA;fluA;fluG;fluG;fluC;fluA;fluC;fluG;fluA;fluG; fluA;fluA;fluA;fluA;fluG;fluG;fluG;fluG;omeU;omeU

3p0Y_6 p;fluU;fluA;fluA;fluG;fluG;fluC;fluA;fluC;fluG;fluA;fluG; fluG;fluA;fluA;fluG;fluG;fluG;fluA;fluA;omeU;omeU

3p0Y_7 p;fluU;fluA;fluA;fluG;fluG;fluC;fluA;fluC;fluG;fluG;fluA; fluA;fluG;fluA;fluA;fluA;fluG;fluG;fluG;omeU;omeU

3p0Y_8 p;fluU;fluA;fluA;fluG;fluG;fluC;fluA;fluC;fluG;fluA;fluA; fluG;fluG;fluA;fluA;fluG;fluA;fluG;fluG;omeU;omeU

3p1Y_1 p;fluU;fluA;fluA;fluG;fluG;fluC;fluA;fluC;fluG;fluA;fluC; fluA;fluA;fluG;fluA;fluA;fluG;fluG;fluG;omeU;omeU

3p1Y_2 p;fluU;fluA;fluA;fluG;fluG;fluC;fluA;fluC;fluG;fluA;fluA; fluG;fluA;fluA;fluG;fluG;fluG;fluC;fluA;omeU;omeU 
3p1Y_3 p;fluU;fluA;fluA;fluG;fluG;fluC;fluA;fluC;fluG;fluA;fluA; fluG;fluC;fluA;fluG;fluA;fluG;fluG;fluA;omeU;omeU

3p1Y_4 p;fluU;fluA;fluA;fluG;fluG;fluC;fluA;fluC;fluG;fluG;fluA; fluG;fluC;fluG;fluG;fluA;fluA;fluA;fluA;omeU;omeU

3p1Y_5 p;fluU;fluA;fluA;fluG;fluG;fluC;fluA;fluC;fluG;fluG;fluA; fluA;fluA;fluG;fluG;fluA;fluG;fluA;fluC;omeU;omeU

3p1Y_6 p;fluU;fluA;fluA;fluG;fluG;fluC;fluA;fluC;fluG;fluA;fluG; fluG;fluA;fluA;fluG;fluC;fluA;fluA;fluG;omeU;omeU

3p1Y_7 p;fluU;fluA;fluA;fluG;fluG;fluC;fluA;fluC;fluG;fluC;fluA; fluA;fluG;fluA;fluG;fluG;fluA;fluG;fluA;omeU;omeU

3p1Y_8 p;fluU;fluA;fluA;fluG;fluG;fluC;fluA;fluC;fluG;fluA;fluA; fluG;fluG;fluA;fluA;fluG;fluC;fluA;fluG;omeU;omeU

3p2Y_1 p;fluU;fluA;fluA;fluG;fluG;fluC;fluA;fluC;fluG;fluG;fluC; fluA;fluG;fluU;fluG;fluG;fluG;fluA;fluA;omeU;omeU

3p2Y_2 p;fluU;fluA;fluA;fluG;fluG;fluC;fluA;fluC;fluG;fluA;fluU; fluG;fluG;fluG;fluG;fluA;fluA;fluG;fluC;omeU;omeU

3p2Y_3 p;fluU;fluA;fluA;fluG;fluG;fluC;fluA;fluC;fluG;fluG;fluU; fluG;fluA;fluC;fluG;fluG;fluG;fluA;fluA;omeU;omeU

3p2Y_4 p;fluU;fluA;fluA;fluG;fluG;fluC;fluA;fluC;fluG;fluG;fluA; fluG;fluG;fluG;fluC;fluA;fluA;fluU;fluG;omeU;omeU

3p2Y_5 p;fluU;fluA;fluA;fluG;fluG;fluC;fluA;fluC;fluG;fluG;fluA; fluU;fluG;fluA;fluA;fluG;fluG;fluC;fluG;omeU;omeU

3p2Y_6 p;fluU;fluA;fluA;fluG;fluG;fluC;fluA;fluC;fluG;fluG;fluG; fluU;fluC;fluG;fluA;fluA;fluG;fluA;fluG;omeU;omeU

3p2Y_7 p;fluU;fluA;fluA;fluG;fluG;fluC;fluA;fluC;fluG;fluG;fluU; fluA;fluG;fluA;fluA;fluG;fluG;fluG;fluC;omeU;omeU

3p2Y_8 p;fluU;fluA;fluA;fluG;fluG;fluC;fluA;fluC;fluG;fluG;fluG; fluA;fluU;fluG;fluA;fluA;fluG;fluC;fluG;omeU;omeU

3p3Y_1 p;fluU;fluA;fluA;fluG;fluG;fluC;fluA;fluC;fluG;fluA;fluA; fluG;fluG;fluG;fluG;fluU;fluU;fluA;fluU;omeU;omeU

3p3Y_2 p;fluU;fluA;fluA;fluG;fluG;fluC;fluA;fluC;fluG;fluG;fluA; fluG;fluU;fluG;fluG;fluA;fluU;fluU;fluA;omeU;omeU

3p3Y_3 p;fluU;fluA;fluA;fluG;fluG;fluC;fluA;fluC;fluG;fluU;fluU; fluA;fluG;fluG;fluG;fluU;fluA;fluG;fluA;omeU;omeU

3p3Y_4 p;fluU;fluA;fluA;fluG;fluG;fluC;fluA;fluC;fluG;fluG;fluG; fluU;fluA;fluG;fluU;fluG;fluA;fluA;fluU;omeU;omeU

3p3Y_5 p;fluU;fluA;fluA;fluG;fluG;fluC;fluA;fluC;fluG;fluG;fluG; fluA;fluA;fluG;fluU;fluU;fluU;fluG;fluA;omeU;omeU

3p3Y_6 p;fluU;fluA;fluA;fluG;fluG;fluC;fluA;fluC;fluG;fluU;fluA; fluG;fluU;fluA;fluA;fluG;fluG;fluG;fluU;omeU;omeU

3p3Y_7 p;fluU;fluA;fluA;fluG;fluG;fluC;fluA;fluC;fluG;fluU;fluU; fluA;fluG;fluG;fluG;fluU;fluA;fluA;fluG;omeU;omeU

3p3Y_8 p;fluU;fluA;fluA;fluG;fluG;fluC;fluA;fluC;fluG;fluG;fluA; fluU;fluU;fluG;fluG;fluU;fluA;fluA;fluG;omeU;omeU

3p4Y_1 p;fluU;fluA;fluA;fluG;fluG;fluC;fluA;fluC;fluG;fluA;fluG; fluA;fluA;fluU;fluU;fluG;fluC;fluG;fluC;omeU;omeU

3p4Y_2 p;fluU;fluA;fluA;fluG;fluG;fluC;fluA;fluC;fluG;fluA;fluG; fluU;fluG;fluG;fluC;fluU;fluA;fluC;fluA;omeU;omeU

3p4Y_3 p;fluU;fluA;fluA;fluG;fluG;fluC;fluA;fluC;fluG;fluG;fluA; fluU;fluA;fluA;fluC;fluU;fluC;fluG;fluG;omeU;omeU

3p4Y_4 p;fluU;fluA;fluA;fluG;fluG;fluC;fluA;fluC;fluG;fluA;fluA; fluC;fluU;fluG;fluA;fluG;fluC;fluG;fluU;omeU;omeU

3p4Y_5 p;fluU;fluA;fluA;fluG;fluG;fluC;fluA;fluC;fluG;fluC;fluU; fluU;fluA;fluG;fluG;fluG;fluA;fluC;fluA;omeU;omeU

3p4Y_6 p;fluU;fluA;fluA;fluG;fluG;fluC;fluA;fluC;fluG;fluG;fluA; fluA;fluU;fluG;fluC;fluU;fluG;fluC;fluA;omeU;omeU

3p4Y_7 p;fluU;fluA;fluA;fluG;fluG;fluC;fluA;fluC;fluG;fluG;fluU; fluC;fluA;fluU;fluA;fluG;fluG;fluC;fluA;omeU;omeU
3p4Y_8 p;fluU;fluA;fluA;fluG;fluG;fluC;fluA;fluC;fluG;fluC;fluU; fluA;fluG;fluA;fluU;fluC;fluG;fluG;fluA;omeU;omeU

3p5Y_1 p;fluU;fluA;fluA;fluG;fluG;fluC;fluA;fluC;fluG;fluG;fluC; fluG;fluC;fluC;fluG;fluG;fluC;fluC;fluG;omeU;omeU

3p5Y_2 p;fluU;fluA;fluA;fluG;fluG;fluC;fluA;fluC;fluG;fluG;fluG; fluG;fluC;fluC;fluG;fluG;fluC;fluC;fluC;omeU;omeU

3p5Y_3 p;fluU;fluA;fluA;fluG;fluG;fluC;fluA;fluC;fluG;fluG;fluC; fluG;fluC;fluG;fluC;fluG;fluG;fluC;fluC;omeU;omeU

3p5Y_4 p;fluU;fluA;fluA;fluG;fluG;fluC;fluA;fluC;fluG;fluG;fluC; fluC;fluC;fluG;fluG;fluG;fluC;fluG;fluC;omeU;omeU

3p5Y_5 p;fluU;fluA;fluA;fluG;fluG;fluC;fluA;fluC;fluG;fluG;fluC; fluC;fluG;fluC;fluG;fluG;fluC;fluC;fluG;omeU;omeU

3p5Y_6 p;fluU;fluA;fluA;fluG;fluG;fluC;fluA;fluC;fluG;fluG;fluG; fluC;fluC;fluG;fluG;fluG;fluC;fluC;fluC;omeU;omeU

3p5Y_7 p;fluU;fluA;fluA;fluG;fluG;fluC;fluA;fluC;fluG;fluC;fluC; fluG;fluG;fluG;fluC;fluC;fluG;fluC;fluG;omeU;omeU

3p5Y_8 p;fluU;fluA;fluA;fluG;fluG;fluC;fluA;fluC;fluG;fluC;fluG; fluC;fluC;fluG;fluG;fluG;fluC;fluC;fluG;omeU;omeU

3p6Y_1 p;fluU;fluA;fluA;fluG;fluG;fluC;fluA;fluC;fluG;fluG;fluC; fluC;fluA;fluG;fluC;fluC;fluU;fluA;fluC;omeU;omeU

3p6Y_2 p;fluU;fluA;fluA;fluG;fluG;fluC;fluA;fluC;fluG;fluC;fluU; fluG;fluC;fluC;fluA;fluC;fluG;fluA;fluC;omeU;omeU

3p6Y_3 p;fluU;fluA;fluA;fluG;fluG;fluC;fluA;fluC;fluG;fluC;fluC; fluA;fluC;fluC;fluA;fluG;fluU;fluC;fluG;omeU;omeU

3p6Y_4 p;fluU;fluA;fluA;fluG;fluG;fluC;fluA;fluC;fluG;fluC;fluA; fluC;fluG;fluA;fluC;fluU;fluC;fluG;fluC;omeU;omeU

3p6Y_5 p;fluU;fluA;fluA;fluG;fluG;fluC;fluA;fluC;fluG;fluC;fluA; fluA;fluU;fluC;fluC;fluC;fluG;fluG;fluC;omeU;omeU

3p6Y_6 p;fluU;fluA;fluA;fluG;fluG;fluC;fluA;fluC;fluG;fluC;fluC; fluC;fluG;fluA;fluU;fluC;fluA;fluG;fluC;omeU;omeU

3p6Y_7 p;fluU;fluA;fluA;fluG;fluG;fluC;fluA;fluC;fluG;fluG;fluC; fluC;fluG;fluA;fluC;fluC;fluU;fluA;fluC;omeU;omeU

3p6Y_8 p;fluU;fluA;fluA;fluG;fluG;fluC;fluA;fluC;fluG;fluG;fluG; fluC;fluC;fluC;fluA;fluA;fluC;fluU;fluC;omeU;omeU

3p7Y_1 p;fluU;fluA;fluA;fluG;fluG;fluC;fluA;fluC;fluG;fluU;fluC; fluG;fluU;fluU;fluA;fluU;fluU;fluG;fluU;omeU;omeU

3p7Y_2 p;fluU;fluA;fluA;fluG;fluG;fluC;fluA;fluC;fluG;fluU;fluG; fluC;fluU;fluG;fluU;fluU;fluU;fluU;fluA;omeU;omeU

3p7Y_3 p;fluU;fluA;fluA;fluG;fluG;fluC;fluA;fluC;fluG;fluU;fluU; fluU;fluG;fluU;fluU;fluU;fluA;fluC;fluG;omeU;omeU

3p7Y_4 p;fluU;fluA;fluA;fluG;fluG;fluC;fluA;fluC;fluG;fluG;fluU; fluC;fluU;fluU;fluA;fluU;fluU;fluU;fluG;omeU;omeU

3p7Y_5 p;fluU;fluA;fluA;fluG;fluG;fluC;fluA;fluC;fluG;fluG;fluU; fluG;fluU;fluU;fluC;fluA;fluU;fluU;fluU;omeU;omeU

3p7Y_6 p;fluU;fluA;fluA;fluG;fluG;fluC;fluA;fluC;fluG;fluU;fluU; fluG;fluU;fluU;fluU;fluA;fluU;fluC;fluG;omeU;omeU

3p7Y_7 p;fluU;fluA;fluA;fluG;fluG;fluC;fluA;fluC;fluG;fluC;fluU; fluG;fluU;fluU;fluG;fluU;fluU;fluA;fluU;omeU;omeU

3p7Y_8 p;fluU;fluA;fluA;fluG;fluG;fluC;fluA;fluC;fluG;fluU;fluG; fluU;fluU;fluU;fluG;fluC;fluA;fluU;fluU;omeU;omeU

3p8Y_1 p;fluU;fluA;fluA;fluG;fluG;fluC;fluA;fluC;fluG;fluU;fluC; fluC;fluA;fluC;fluG;fluC;fluC;fluU;fluC;omeU;omeU

3p8Y_2 p;fluU;fluA;fluA;fluG;fluG;fluC;fluA;fluC;fluG;fluC;fluU; fluA;fluC;fluC;fluG;fluU;fluC;fluC;fluC;omeU;omeU

3p8Y_3 p;fluU;fluA;fluA;fluG;fluG;fluC;fluA;fluC;fluG;fluU;fluG; fluC;fluU;fluC;fluC;fluC;fluA;fluC;fluC;omeU;omeU

3p8Y_4 p;fluU;fluA;fluA;fluG;fluG;fluC;fluA;fluC;fluG;fluC;fluC; fluC;fluU;fluC;fluC;fluC;fluU;fluG;fluA;omeU;omeU 
3p8Y_5 p;fluU;fluA;fluA;fluG;fluG;fluC;fluA;fluC;fluG;fluC;fluU; fluG;fluC;fluU;fluC;fluC;fluA;fluC;fluC;omeU;omeU

3p8Y_6 p;fluU;fluA;fluA;fluG;fluG;fluC;fluA;fluC;fluG;fluC;fluC; fluU;fluA;fluU;fluC;fluC;fluG;fluC;fluC;omeU;omeU

3p8Y_7 p;fluU;fluA;fluA;fluG;fluG;fluC;fluA;fluC;fluG;fluC;fluA; fluU;fluU;fluC;fluG;fluC;fluC;fluC;fluC;omeU;omeU

3p8Y_8 p;fluU;fluA;fluA;fluG;fluG;fluC;fluA;fluC;fluG;fluC;fluG; fluC;fluC;fluC;fluU;fluC;fluC;fluU;fluA;omeU;omeU

3p9Y_1 p;fluU;fluA;fluA;fluG;fluG;fluC;fluA;fluC;fluG;fluU;fluU; fluC;fluU;fluU;fluU;fluC;fluG;fluC;fluC;omeU;omeU

3p9Y_2 p;fluU;fluA;fluA;fluG;fluG;fluC;fluA;fluC;fluG;fluC;fluG; fluC;fluU;fluU;fluC;fluU;fluC;fluU;fluU;omeU;omeU

3p9Y_3 p;fluU;fluA;fluA;fluG;fluG;fluC;fluA;fluC;fluG;fluU;fluU; fluC;fluU;fluC;fluG;fluU;fluC;fluU;fluC;omeU;omeU

3p9Y_4 p;fluU;fluA;fluA;fluG;fluG;fluC;fluA;fluC;fluG;fluU;fluC; fluC;fluU;fluU;fluG;fluC;fluU;fluU;fluC;omeU;omeU

3p9Y_5 p;fluU;fluA;fluA;fluG;fluG;fluC;fluA;fluC;fluG;fluU;fluU; fluU;fluC;fluG;fluU;fluC;fluU;fluC;fluC;omeU;omeU

3p9Y_6 p;fluU;fluA;fluA;fluG;fluG;fluC;fluA;fluC;fluG;fluC;fluU; fluU;fluU;fluC;fluC;fluG;fluU;fluU;fluC;omeU;omeU

3p9Y_7 p;fluU;fluA;fluA;fluG;fluG;fluC;fluA;fluC;fluG;fluU;fluU; fluU;fluG;fluC;fluC;fluU;fluC;fluC;fluU;omeU;omeU

3p9Y_8 p;fluU;fluA;fluA;fluG;fluG;fluC;fluA;fluC;fluG;fluC;fluC; fluU;fluU;fluG;fluU;fluU;fluC;fluC;fluU;omeU;omeU

3p10Y_1 p;fluU;fluA;fluA;fluG;fluG;fluC;fluA;fluC;fluG;fluU;fluC; fluC;fluC;fluU;fluU;fluC;fluU;fluU;fluC;omeU;omeU

3p10Y_2 p;fluU;fluA;fluA;fluG;fluG;fluC;fluA;fluC;fluG;fluU;fluC; fluU;fluC;fluU;fluU;fluC;fluC;fluC;fluU;omeU;omeU

3p10Y_3 p;fluU;fluA;fluA;fluG;fluG;fluC;fluA;fluC;fluG;fluC;fluU; fluC;fluU;fluU;fluC;fluU;fluC;fluU;fluC;omeU;omeU

3p10Y_4 p;fluU;fluA;fluA;fluG;fluG;fluC;fluA;fluC;fluG;fluC;fluU; fluU;fluC;fluC;fluU;fluC;fluU;fluC;fluU;omeU;omeU

3p10Y_5 p;fluU;fluA;fluA;fluG;fluG;fluC;fluA;fluC;fluG;fluU;fluC; fluC;fluC;fluC;fluU;fluU;fluU;fluU;fluC;omeU;omeU

3p10Y_6 p;fluU;fluA;fluA;fluG;fluG;fluC;fluA;fluC;fluG;fluC;fluC; fluU;fluC;fluU;fluU;fluU;fluU;fluC;fluC;omeU;omeU

3p10Y_7 p;fluU;fluA;fluA;fluG;fluG;fluC;fluA;fluC;fluG;fluC;fluU; fluC;fluC;fluC;fluU;fluU;fluU;fluC;fluU;omeU;omeU

3p10Y_8 p;fluU;fluA;fluA;fluG;fluG;fluC;fluA;fluC;fluG;fluC;fluC; fluU;fluC;fluC;fluU;fluU;fluC;fluU;fluU;omeU;omeU

\section{SUPPLEMENTAL MATERIAL}

Supplemental material is available for this article.

\section{ACKNOWLEDGMENTS}

We thank Rick Sidler, Mark Levorse, and the oligonucleotide synthesis team. We also thank our colleagues at Sirna-Merck for their support over the past three years.

Received November 9, 2011; accepted June 22, 2012.

\section{REFERENCES}

Bartel DP. 2009. MicroRNAs: Target recognition and regulatory functions. Cell 136: 215-233.

Bramsen JB, Kjems J. 2011. Chemical modification of small interfering RNA. Methods Mol Biol 721: 77-103.

Bramsen JB, Laursen MB, Damgaard CK, Lena SW, Babu BR, Wengel J, Kjems J. 2007. Improved silencing properties using small internally segmented interfering RNAs. Nucleic Acids Res 35: 58865897.

Chorn G, Zhao L, Sachs AB, Flanagan WM, Lim LP. 2010. Persistence of seed-based activity following segmentation of a microRNA guide strand. RNA 16: 2336-2340.

Chu CY, Rana TM. 2008. Potent RNAi by short RNA triggers. RNA 14: $1714-1719$.

Crooks GE, Hon G, Chandonia JM, Brenner SE. 2004. WebLogo: A sequence logo generator. Genome Res 14: 1188-1190.

Elbashir SM, Harborth J, Lendeckel W, Yalcin A, Weber K, Tuschl T. 2001. Duplexes of 21-nucleotide RNAs mediate RNA interference in cultured mammalian cells. Nature 411: 494-498.

Haringsma HJ, Li JJ, Soriano F, Kenski DM, Flanagan WM, Willingham AT. 2012. mRNA knockdown by single strand RNA is improved by chemical modifications. Nucleic Acids Res 40: 4125-4136.

Hatziapostolou M, Polytarchou C, Aggelidou E, Drakaki A, Poultsides GA, Jaeger SA, Ogata H, Karin M, Struhl K, Hadzopoulou-Cladaras $\mathrm{M}$, et al. 2011. An HNF4 $\alpha$-miRNA inflammatory feedback circuit regulates hepatocellular oncogenesis. Cell 147: 1233-1247.

Henry JC, Azevedo-Pouly AC, Schmittgen TD. 2011. microRNA replacement therapy for cancer. Pharm Res 28: 3030-3042.

Jackson AL, Burchard J, Leake D, Reynolds A, Schelter J, Guo J, Johnson JM, Lim L, Karpilow J, Nichols K, et al. 2006. Positionspecific chemical modification of siRNAs reduces "off-target" transcript silencing. RNA 12: 1197-1205.

Kim VN, Han J, Siomi MC. 2009. Biogenesis of small RNAs in animals. Nat Rev Mol Cell Biol 10: 126-139.

Lim LP, Lau NC, Garrett-Engele P, Grimson A, Schelter JM, Castle J, Bartel DP, Linsley PS, Johnson JM. 2005. Microarray analysis shows that some microRNAs downregulate large numbers of target mRNAs. Nature 433: 769-773.

Manoharan M, Akinc A, Pandey RK, Qin J, Hadwiger P, John M, Mills K, Charisse K, Maier MA, Nechev L, et al. 2011. Unique gene-silencing and structural properties of $2^{\prime}$-fluoro-modified siRNAs. Angew Chem Int Ed Engl 50: 2284-2288.

Martinez J, Patkaniowska A, Urlaub H, Luhrmann R, Tuschl T. 2002. Single-stranded antisense siRNAs guide target RNA cleavage in RNAi. Cell 110: 563-574.

Raal FJ, Santos RD, Blom DJ, Marais AD, Charng MJ, Cromwell WC, Lachmann RH, Gaudet D, Tan JL, Chasan-Taber S, et al. 2010. Mipomersen, an apolipoprotein B synthesis inhibitor, for lowering of LDL cholesterol concentrations in patients with homozygous familial hypercholesterolaemia: A randomised, double-blind, placebo-controlled trial. Lancet 375: 998-1006.

Schwarz DS, Hutvagner G, Haley B, Zamore PD. 2002. Evidence that siRNAs function as guides, not primers, in the Drosophila and human RNAi pathways. Mol Cell 10: 537-548.

Sun X, Rogoff H, Li CJ. 2008. Asymmetric RNA duplexes mediate RNA interference in mammalian cells. Nat Biotechnol 26: 1379-1382.

Swayze EE, Prakash TP, Lima W, Crooke S. 2011. Activation of RNA interference in animals with single stranded oligonucleotides. 7th Annual Meeting of the Oligonucleotide Therapeutics Society, Copenhagen, Denmark. 

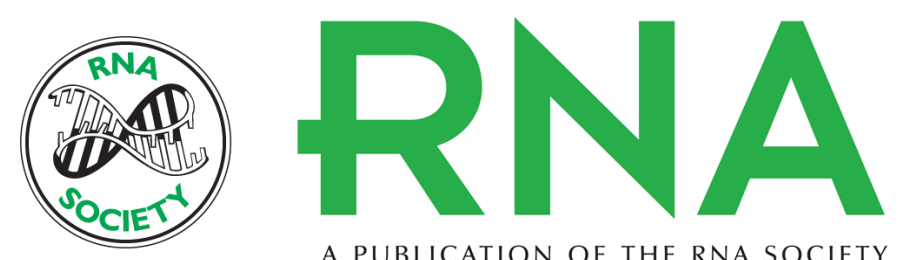

A PUBLICATION OF THE RNA SOCIETY

\section{Single-stranded microRNA mimics}

Guillaume Chorn, Molly Klein-McDowell, Lihong Zhao, et al.

RNA 2012 18: 1796-1804 originally published online August 21, 2012

Access the most recent version at doi:10.1261/rna.031278.111

Supplemental

Material

References This article cites 18 articles, 4 of which can be accessed free at:

http://rnajournal.cshlp.org/content/18/10/1796.full.html\#ref-list-1

\section{License}

Email Alerting Service

http://rnajournal.cshlp.org/content/suppl/2012/07/25/rna.031278.111.DC1

top right corner of the article or click here.

To subscribe to $R N A$ go to:

http://rnajournal.cshlp.org/subscriptions 